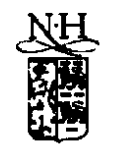

ELSEVIER

\title{
Monte Carlo simulation of layered high-temperature superconductors
}

\author{
A.R. Price ${ }^{a}$, S.J. Cox ${ }^{a}$, H. Fangohr ${ }^{a b}$ and P.A.J. de Groot ${ }^{b}$ \\ 'Department of Electronics and Computer Science, and \\ b Department of Physics and Astronomy, \\ University of Southampton, Highfield, Southampton, Hampshire SO17 1BJ, United Kingdom
}

We present results from Monte Carlo simulations of the vortex state in layered high temperature superconductors. We use a set of potentials derived from the Lawrence-Doniach free-energy functional which incorporates (i) intra-layer coupling (ii) inter-layer Josephson and electromagnetic interactions. We have employed a novel technique for performing an in-plane infinite lattice summation for the intra-layer interactions. This provides a minimum 50,000 times faster speed-up in the simulations over previous naive methods which add periodic images in shells of increasing radius. We present results of the numerical B-T phase diagram in the pure system and obtain good agreement with available experimental/theoretical results.

\section{INTRODUCTION}

The nature of the vortex state in high temperature superconductors is not yet fully understood. The competition between the ordering forces of the elastic lattice and the disordering influence of thermal energy and material pinning leads to a number of possible vortex phases including a Bragg glass, a vortex glass and liquid states. We report some preliminary results from Monte Carlo simulations of the vortex state in a model threedimensional system.

\section{MODEL}

We perform particle-particle simulations using a model derived from the Lawrence-Doniach free energy functional. We consider $N_{z}$ superconducting layers separated by a distance $s$ and each containing $N_{x y}$ point-like vortex pancakes. Each vortex is described by its position vector $\boldsymbol{r}_{i, k}$ within the plane and vortex lines are defined by connecting each vortex to a unique neighbour in the layers above and below. Periodic boundary conditions are applied in all directions. Each layer has an oblique geometry commensurate with the hexatic nature of the solid phase. The vortex density is fixed such that $N_{x y} / A_{x y}=$ $\Phi_{d} B$ giving a trigonal lattice spacing of $a_{0}{ }^{2}=$ $(4 / 3)^{1 / 2}\left(\Phi_{d} B\right)$, where $\Phi_{0}=h c / 2 e$ is the flux quantum.

We apply an intra-layer interaction potential acting between all vortices in the same layer. This takes the form of a modified Bessel function appropriate to line-like interactions in the vortex state [1]. In contrast to previous numerical simulations we apply a full set of potentials between the pancakes that define a single line, including Josephson pairwise, three and four body interactions and electromagnetic contributions. These are derived in [2].

The scaling length $\lambda$ for the intra-layer interaction is large and yields a long-range potential. We have developed a new summation method to determine the system energy summed over infinite periodic repeats of the unit cell. The Bessel function is expanded using the Gegenbauer addition formulae [3]. The potential between a pair of particles is expressed as:

$K_{0}^{*}\left(\frac{w}{\lambda}\right)=K_{0}\left(\frac{z}{\lambda}\right)+\sum_{k=-\infty}^{\infty} I_{k}\left(\frac{z}{\lambda}\right)\left[c_{k} \cos (k \theta)-s_{k} \sin (k \theta)\right]$

where $c_{k}$ and $s_{k}$ are coefficients determining the energy contribution from the infinite lattice, which can be pre-computed. $z$ and $\theta$ refer to unit cell separations and $I_{k}$ is a modified Bessel function. The resulting calculation is a minimum of 50,000 times faster than the naive summation of image cells at increasing radius (see Figure 1).

Various observables are used to determine the phase of the vortex system. These include Delaunay triangulation, hexatic order parameter, and number of topological defects (definitions as in e.g. [4]). To probe the translational properties of the system 


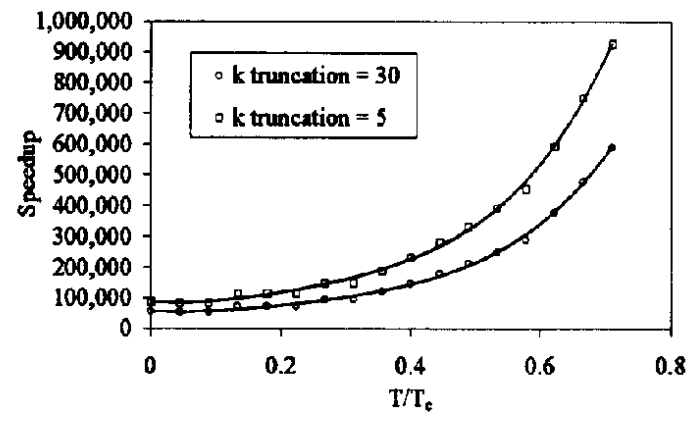

Figure 1. Speedup achieved by infinite lattice summation over naive summation method as a function of temperature in the simulation and the number of coefficients used in (1).

we calculate the structure factor from the twodimensional Fourier transform of the ensemble average of the vortex positions at a given temperature.

\section{RESULTS}

With material parameters appropriate to BSCCO compounds $\left(\lambda_{a b}=1500 \AA, s=10 \AA, \Gamma=500\right)$ we have performed Monte Carlo simulations on systems of size $N_{x y}=192$ and $N_{z}=10$. Using $1 \times 10^{4}$ sweeps to equilibrate and $5 \times 10^{4}$ sweeps for observations we have determined the phase diagram of the pure three dimensional system using field cooled annealing. The relatively low number of sweeps employed leads to some hysteretic effects in the melting properties of the system and for consistency we present results from annealing runs only.

Starting from a melted state we have reduced the temperature in $0.25 \mathrm{~K}$ steps through the freezing point. We identify a solid phase through a sharp increase in hexatic order, a decrease in the number of topological defects and the onset of Bragg peaks in the structure factor. By monitoring the specific heat capacity of the system energy we identify the temperature at which freezing occurs. The results are presented in figure 2. A theoretical melting line based on nearest neighbour interactions is given by [1]:

$$
B_{m}=\frac{c_{L}^{4} \Phi_{0}^{3}}{\left(\mu_{0} \pi\right)^{2} \Gamma^{2}\left(k_{s} T\right)^{2} \lambda_{a b}^{4}} .
$$

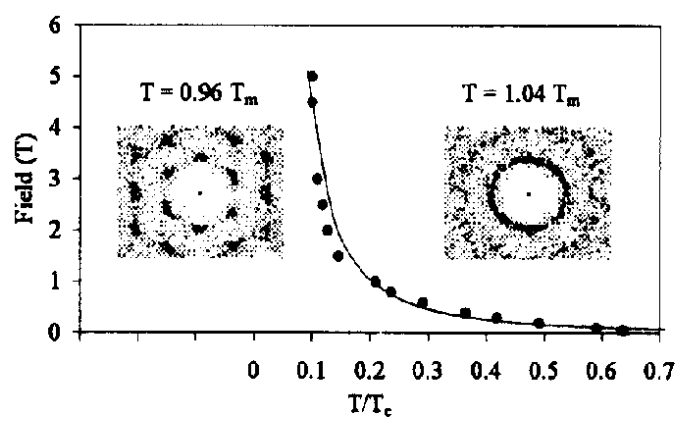

Figure 2. Numerical phase diagram obtained from annealing data. Trend line is fitted from equation (2) Inset: Structure factors observed just above and below the melting temperature $T_{m}$.

The line in figure 2 is fitted using an optimal value of $c_{L}=0.27$. This is similar to empirical results [5].

Pinning is introduced into the model system as a periodic disordering energy surface superposed over the simulation cell. Qualitatively the surface is generated such that typically five maxima and minima occur within each vortex spacing of the lattice at 1T. At constant field, the freezing point of the vortex system is observed to reduce in the presence of pinning and decreases as the average pinning strength increases as expected.

Our simulations indicate that use of the full set of interaction potentials yields results similar to those obtained with simplified potentials. Further, as discussed in [3], our infinitely summed potential does not introduce unphysical defects into the annealed state and therefore pinned systems can be simulated more reliably. We will report on further results of such simulations elsewhere.

\section{REFERENCES}

1. Tinkham, M., Introduction to Superconductivity, Second edition, (1996).

2. Krämer, C., Physica C, 256 (1996) 236-244.

3. Fangohr, H., Price, A.R., Cox, S.J., de Groot, P.A.J. and Daniell, G.J., Submitted to J. Comp. Phys., (1999).

4. Ryu, S. and Stroud, D., Phys. Rev. B, 54 (1996) 1320-1333.

5. Blatter, G., Feigel'man, M.V., Geshkenbein, V.B., Larkin, A.I. and Vinokur, V.M., Rev. Mod. Phys., 66 (1994) 1125-1388. 